\title{
Blocking Probabilities of Optical Burst Switching Networks Based on Reduced Load Fixed Point Approximations
}

\author{
Zvi Rosberg*, Hai Le $\mathrm{Vu}^{\dagger}$, Moshe Zukerman ${ }^{\dagger}$ and Jolyon White ${ }^{\dagger}$ \\ * Department of Communication Systems Engineering Ben Gurion University, Beer-Sheva, 84105, Israel \\ Email: rosberg@bgumail.bgu.ac.il \\ This research has been conducted while Z. Rosberg was visiting \\ the ARC Special Research Center for Ultra-Broadband Information Networks, University of Melbourne. \\ $\dagger$ ARC Special Research Center for Ultra-Broadband Information Networks \\ Department of E\&E Engineering, The University of Melbourne, Melbourne, VIC 3010, Australia \\ Email: h.vu@ee.mu.oz.au; m.zukerman@ee.mu.oz.au; j.white@ee.mu.oz.au \\ M. Zukerman is visiting the Department of Electronic Engineering, \\ City University of Hong Kong, between November 2002 and July 2003.
}

\begin{abstract}
This paper provides a framework for analysis and performance evaluation of Optical Burst Switching (OBS) networks. In particular, a new reduced load fixed point approximation model to evaluate blocking probabilities in OBS networks is introduced. The model is versatile enough to cover known OBS reservation policies such as Just-Enough-Time (JET), Just-InTime (JIT), Burst Segmentation and Route-dependent Priorities. The accuracy of the model is confirmed by simulation and the various policies are compared.
\end{abstract}

\section{INTRODUCTION}

Recent technology advancements in optical packet switching [5], [12] and [17] give rise to the need for performance evaluation methodologies that will support business decisions on technology migration, network dimensioning and Service Level Agreements (SLAs).

A preferred option for optical packet switching systems is Optical Burst Switching (OBS) (see [10], [12], [15], [17] and [18]) that has been proposed as an efficient switching technique to exploit the terabit bandwidth of Wavelength Division Multiplexing (WDM) transmission technology.

In OBS, IP packets with a common destination arriving at the same ingress node are aggregated into large bursts, each being switched and routed as one unit. OBS can reduce the switching (processing and fabric reconfiguration) since only one header and possibly a trailer are associated with each burst. In OBS the header (also called label or control packet) precedes the burst payload and attempts to reserve the required switching and transmission resources at each switch and output link port along the route. The header can be transmitted either on the payload wavelength, or on one or more separate control wavelengths. The payload follows the header without waiting for acknowledgment. At every switch, if the requested resources are available, the burst is transparently switched to its next hop; otherwise, the burst is blocked and a fraction or all of it is lost.

OBS is motivated by the need to exploit and better utilize lightpath transmission capacities. Given that current optical transmission rate on a single fibre is of the order of Tbit/s ([5]), while switching capacity (electronically or optically) is in the order of $10 \mathrm{~Gb} / \mathrm{s}$ ([5], [12] and [17]), OBS can nevertheless achieve $\mathrm{Tb} / \mathrm{s}$ end-to-end communication channels for IP packets. The chief reason is that only a very small portion of each burst (header and possibly trailer, see e.g., [17]) is subject to switching while its main part (payload) is transmitted on an end-to-end lightpath.

Note that while optical micro-elecro-mechanical switches (MEMS) can only provide fabric reconfiguration time in the order of a few milliseconds, Semiconductor Optical Amplifiers (SOAs) achieve reconfiguration time in the order of a few nanoseconds. More recent techniques [6] even achieve reconfiguration time in the order of a few hundred picoseconds. The two latter technologies enable OBS switches to operate at $10 \mathrm{~Gb} / \mathrm{s}$. It is worth noting that devices capable of reconfiguration time in the order of a few picoseconds [22] are already being used in research laboratories, and are expected to enable OBS switches to operate at $40 \mathrm{~Gb} / \mathrm{s}$.

We assume here that all switches support full wavelength conversion, whereby a burst can use any available wavelength at each link along its route. The cases of no and limited wavelength conversion [21] has been studied separately and the results are reported in [13].

OBS differs from standard circuit switching in two main aspects. Firstly, OBS bursts immediately follow their request headers without waiting for a reservation acknowledgement. Since buffering in optical switches is not practical, bursts may use bandwidth resources along several links and still be blocked and lost without completing their routes. In circuit switching, on the other hand, transmission starts only after 
an end-to-end path reservation is acknowledged. Secondly, in circuit switching, allocated resources are kept throughout the end-to-end transmission, while in OBS, the reserved resources at each switch and output link port are held only for the duration they are needed.

OBS is also different from packet switching since OBS bursts are not buffered at the switches, while in packet switching they are. Furthermore, due to reservations, OBS bursts use the path links in a time-synchronized manner, while packets use them asynchronously. It is worth noting that in OBS networks, where link propagation delay is significantly larger than burst transmission time, multiple bursts could simultaneously propagate not only along the same route but also along the same link and wavelength [4].

Several OBS reservation protocols have been proposed by researchers. In this paper we consider the following key proposals.

- A variation of the Just-In-Time (JIT) protocol [18], whereby the reservation (control) packet is not aware of the burst length and reserves the relevant link bandwidth (if available) for the entire burst as soon as it arrives at the switch. The variation we consider is without acknowledgement as the maximal burst size is typically small compared with the link transmission capacity.

- The Just-Enough-Time (JET) protocol [10], whereby the control packet is aware of the burst length and reserves the link bandwidth (if available) for the burst duration starting from the predetermined time offset.

- The Burst Segmentation [3] and [15], whereby the reservation (control) packet is aware of the burst length and reserves the wavelength (bandwidth) starting from the first moment it becomes available until the remainder of the burst is transmitted. In this case, the initial part of the burst until the required wavelength on the output fibre becomes free, is discarded and the remainder of the burst is successfully transmitted.

- Preemptive priority burst service policies in each switching node, whereby the priority depends on the route a burst follows [19].

It is well accepted after almost two decades of extensive studies that exact solutions for a blocking network of the type presented above are unattainable. Even in network models that have a product form solution (e.g., conventional circuit switched networks) their computational complexity is NPcomplete [8].

The difficulties related to network models have lead OBS researchers to use a single link model that may provide crude upper bounds for the link blocking probability [14]. Furthermore, the single node analyses are limited by not considering network related issues such as the following two contrasting effects:

- The increased load caused by eventually unsuccessful bursts along their paths before they are discarded.

- The reduced load resulted from blocking of bursts.

These limitations motivate our network modelling approach by which we can investigate and compare between blocking probabilities of various OBS reservation policies and further study the effects of burst routing, burst admission control and other networking aspects. In this paper, we rely on the socalled reduced load fixed point approximation whereby each link blocking probability is approximated by considering only the reduced offered load caused by the blocking on other links in the network.

The remainder of the paper is organized as follows. In Section II we present in detail the reduced load fixed point approximation. In Section III we show how the approximation is applied to various OBS reservation policies such as JET, JIT, Burst Segmentation and Route-Dependent Priorities. In Section IV we validate the approximation by simulation and in Section $\mathrm{V}$ we compare between the various policies by using our approximation method. Finally, the conclusions are presented in Section VI.

\section{The Reduced LoAd Fixed Point Approximation}

In this section we describe a unified model we propose as a fast computational tool to evaluate blocking probabilities of various OBS policies in a network. The model attempts to capture the major elements by which various policies are distinguished. Secondary elements that are marginal in our opinion, are left out in this paper but can be incorporated rather easily.

Consider a network with $J$ directional links labelled by $1,2, \ldots, J$, and suppose that link $j$ comprises $F_{j}$ optical fibers. Each optical fibre is capable to transmit data only in one fixed direction and serves up to $W$ concurrent logical channels using WDM. Let $N_{j}$ be the number of concurrent logical transmission channels, hereinafter wavelength channels, in link $j$ in a given direction. Thus, $N_{j}=W \cdot F_{j}$.

A route $\boldsymbol{r}=\left(r_{1}, r_{2}, \ldots, r_{n}\right)$ of length $n$ (i.e., with $n$ hops) is an ordered set of links that connect the source node to the destination node. Let $\mathcal{R}$ be the set of all possible routes. We assume that bursts offered to route $r$ arrive according to a Poisson process with rate $\lambda \boldsymbol{r}$ and all arrival processes are independent.

A burst offered to route $r$ uses a single wavelength channel from each link along the route until the first link where it is being blocked or until it exits the network. That is, if the burst is first blocked by link $r_{j}$ it uses a single wavelength channel from links $r_{1}, r_{2}, \ldots, r_{j-1}$ at subsequent time intervals that are shifted by the propagation delays of each subsequent link. We further assume that burst transmission times are independent and exponentially distributed and permit different bandwidth capacities on the links. Thus, we denote by $\mu_{j}$ the transmission rate of link $j$.

Typically, maximal burst size is small compared with the link transmission capacity and therefore we assume that at any instant a burst occupies no more than one link. A burst is in progress along a route $\boldsymbol{r}$ at time $t$ if it uses at least one wavelength channel along that route at time $t$. Accordingly, a burst may be in progress even when some of the wavelength channels along its route are used by other bursts. Let $X_{\boldsymbol{r}}(t)$ be 
the number of bursts on route $\boldsymbol{r}$ at time t, and $\boldsymbol{Y}_{\boldsymbol{r}}(t)$ a vector of size $X_{\boldsymbol{r}}(t)$ in which every element in the vector represents the time elapsed from the moment that the corresponding burst has finished its transmission on the first wavelength channel on its route (namely $r_{1}$ ), until time $t$. If the burst still uses a wavelength channel in $r_{1}$, then this element value is zero. Suppose that at every OBS switch, bursts are allocated resources in the order of their reservation request arrivals.

Since subsequent wavelength channels of each burst are used at fixed time offsets and the transmission times are exponential (memoryless), the process $\left\{\left(X_{\boldsymbol{r}}(t), \boldsymbol{Y}_{\boldsymbol{r}}(t)\right) \mid \boldsymbol{r} \in \mathcal{R}, t \geq 0\right\}$ is a Markov process. Moreover, since $X_{\boldsymbol{r}}(t)$ takes a finite number of values and $\boldsymbol{Y}_{\boldsymbol{r}}(t)$ evolves linearly in time and varies within a bounded interval, the process has a stationary distribution.

Our aim is to evaluate the stationary blocking probability of an arbitrary burst and of a burst that is offered to route $r$. Given the difficulty in obtaining exact mathematical results for this problem, we assume as in [1], [7], [14] and [20] that each blocking event occurs independently from link to link along any route.

As will become apparent, the independence assumption enables us to decouple between the network links but still takes into account the reduced offered load resulting from blocking. It is the most crucial assumption we make in our approximation model and therefore we verify it by simulation. Note that other assumptions made in our model only have secondary effects and can be relaxed once the independence assumption above is proved valid. For instance, the Poisson arrivals that leads to an Erlang formula for the blocking probability along each route link, can be replaced with another distribution for which the blocking probability is attainable. This is certainly a subject for further research.

Consider the process $\left\{\left(X_{\boldsymbol{r}}(t), \boldsymbol{Y}_{\boldsymbol{r}}(t)\right) \mid \boldsymbol{r} \in \mathcal{R}, t \geq 0\right\}$ under stationary condition and let $\boldsymbol{B}=\left(B_{1}, B_{2}, \ldots, B_{J}\right)$ denote the vector of stationary link blocking probabilities. Under the independence assumption above, it follows that the load offered to link $j, \rho_{j}$, satisfies

$$
\rho_{j}=\mu_{j}^{-1} \sum_{\boldsymbol{r} \in \mathcal{R}} \lambda \boldsymbol{r} \prod_{i=1}^{J}\left(1-I(i, j, \boldsymbol{r}) \cdot B_{i}\right),
$$

where $I(i, j, \boldsymbol{r})$ equals 1 or 0 depending whether or not $i, j \in \boldsymbol{r}$ and link $i$ strictly precedes link $j$ along route $\boldsymbol{r}$, respectively.

Furthermore, the independence assumption implies that the offered load to each link $j$ is a Poisson process with rate $\rho_{j}$. Thus, the blocking probability is given by the following Erlang Formula [2]:

$$
B_{j} \stackrel{\text { def }}{=} E\left(\rho_{j}, N_{j}\right)=\frac{\rho_{j}^{N_{j}} / N_{j} !}{\sum_{k=0}^{N_{j}} \rho_{j}^{k} / k !} .
$$

Combining equations (1) and (2) yields the following Erlang Fixed Point (EFP) equations satisfied by the approximate link blocking probabilities,

$$
B_{j}=E\left(\mu_{j}^{-1} \sum_{\boldsymbol{r} \in \mathcal{R}} \lambda \boldsymbol{r} \prod_{i=1}^{J}\left(1-I(i, j, \boldsymbol{r}) \cdot B_{i}\right), N_{j}\right) .
$$

Notice that the EFP equations impose consistency among the link blocking probabilities and their respective offered loads under the link independence assumption.

Resolving the vector $\boldsymbol{B}$ from the EFP equations (3) and invoking the independence assumption again, the approximate blocking probability of bursts offered to route $\boldsymbol{r}, B(\boldsymbol{r})$, satisfies

$$
B(\boldsymbol{r})=1-\prod_{i \in \boldsymbol{r}}\left(1-B_{i}\right),
$$

and the blocking probability of an arbitrary burst $B$, satisfies

$$
B=\frac{1}{\Lambda} \sum_{\boldsymbol{r} \in \mathcal{R}} \lambda_{\boldsymbol{r}} \cdot B(\boldsymbol{r}),
$$

where $\Lambda=\sum_{\boldsymbol{r} \in \mathcal{R}} \lambda \boldsymbol{r}$.

Notice that the OBS reduced load offered to link $j$ as given in equation (1) is larger than $\mu_{j}^{-1} \sum_{\boldsymbol{r} \in \mathcal{R}} \lambda \boldsymbol{r} \prod_{i \in \boldsymbol{r} \backslash\{j\}}\left(1-B_{i}\right)$, the reduced load offered to link $j$ in a conventional circuit switching network.

The most efficient way to solve the link blocking probabilities (if a solution exists) is by the following successive substitution procedure. For any given vector of blocking probabilities $\boldsymbol{B}$ define the transformation vector $\boldsymbol{T}(\boldsymbol{B}) \stackrel{\text { def }}{=}\left(T_{1}(\boldsymbol{B}), \ldots, T_{J}(\boldsymbol{B})\right)$ by

$$
T_{j}(\boldsymbol{B})=E\left(\mu_{j}{ }^{-1} \sum_{\boldsymbol{r} \in \mathcal{R}} \lambda \boldsymbol{r} \prod_{i=1}^{J}\left(1-I(i, j, \boldsymbol{r}) \cdot B_{i}\right), N_{j}\right) .
$$

We begin the successive substitution procedure with a certain initial blocking probability vector $\boldsymbol{B}$ and repeatedly apply the transformation $\boldsymbol{T}(\boldsymbol{B})$. That is, we compute $\boldsymbol{B}^{n}=\boldsymbol{T}\left(\boldsymbol{B}^{n-1}\right)$ for $n=1,2, \ldots$ (where $\boldsymbol{B}^{0}=\boldsymbol{B}$ ), until $\boldsymbol{B}^{n}$ is sufficiently close to $\boldsymbol{B}^{n-1}$.

Observe that the transformation $\boldsymbol{T}(\boldsymbol{B})$ is a continuous mapping from the compact set $[0,1]^{J}$ to itself and therefore it has a fixed point by the Brouwer fixed-point theorem [9]. We cannot establish the uniqueness of the solutions as has been established in [7] for the conventional circuit switching network. Nevertheless, in all the numerical examples we used for this study, the iterations always converge to a unique fixed point. The following convergence properties though, can be established.

Since the transformation $\boldsymbol{T}(\boldsymbol{B})$ is decreasing, namely, $\boldsymbol{T}(\boldsymbol{B})<\boldsymbol{T}\left(\boldsymbol{B}^{\prime}\right)$ whenever $\boldsymbol{B}>\boldsymbol{B}^{\prime}$, the following result is easily derived (as done in [20] for the circuit switching network).

Theorem 1: Starting with $\boldsymbol{B}^{0}=\mathbf{1}$ (the vector of all ones), then the successive substitution for every $n$ yields the following upper and lower bounds on all fixed point solutions $B^{\star}=\boldsymbol{T}\left(B^{\star}\right)$ :

$$
\hat{\boldsymbol{B}}=\boldsymbol{B}^{1}<\boldsymbol{B}^{2 n+1}<\boldsymbol{B}^{2 n+3}<\boldsymbol{B}^{\star},
$$


and

$$
\boldsymbol{B}^{\star}<\boldsymbol{B}^{2 n+2}<\boldsymbol{B}^{2 n}<\boldsymbol{B}^{0}=\mathbf{1},
$$

where $\hat{\boldsymbol{B}}=\left(\hat{B}_{1}, \hat{B}_{2}, \ldots, \hat{B}_{J}\right)$, and $\hat{B}_{j}$ is the blocking probability resulting from the total exogenous arrival rates to link $j$. Furthermore, the sequence $\left\{\boldsymbol{B}^{2 n}\right\}$ converges to an upper bound $\bar{B}$, and the sequence $\left\{\boldsymbol{B}^{2 n+1}\right\}$ converges to a lower bound $\underline{B}$ on every fixed point solution.

Observe that since the transformation $\boldsymbol{T}(\boldsymbol{B})$ is decreasing it follows that $\underline{B}=\boldsymbol{T}(\overline{\boldsymbol{B}})$ and $\overline{\boldsymbol{B}}=\boldsymbol{T}(\underline{\boldsymbol{B}})$. Therefore, if the lower and the upper bounds are not sufficiently close then one may continue the iterations from some convex combination of both vectors. Restarting from any convex combinations will drive the iterations to the fixed point solutions. As mentioned above, in all the examples used for this study, the iterations always converge to a unique fixed point.

The Erlang formula in the EFP equations (3) can be replaced with other blocking probabilities as explained in Section III. For those cases, convergence to the upper and lower bounds is also guaranteed so long as the transformation $\boldsymbol{T}(\boldsymbol{B})$ is decreasing.

\section{Extensions AND ApPLICATIONS}

As discussed above, the Erlang fixed point equations and the successive substitution iterations can be applied to the JIT variant and the JET policies, and be extended to other policies. Below we show how to apply them to JET and JIT and derive the equations for policies that allow burst preemption and burst segmentation.

\section{A. JET and JIT Policies}

The formulae obtained in Section II can immediately be applied to the JIT and the JET regimes. With JET, the transmission rate $\mu_{j}$ at link $j$ is set to $1 / E\left(S_{j}\right)$, where $S_{j}$ is the actual burst service time in link $j$. With JIT, we set $\mu_{j}=1 /\left(E\left(S_{j}\right)+E\left(D_{j}\right)\right)$, where $D_{j}$ is the time offset between the arrivals of the control packet and its corresponding burst to the switching node of link $j$. Otherwise, (1)-(3) are left intact.

\section{B. Burst Segmentation}

Since an OBS burst is an aggregation of many IP packets, one can significantly reduce the packet loss probability in OBS networks by applying a technique called Optical Composite Burst Switching (OCBS) [3] also called Burst Segmentation [15] that discards only the initial part of a burst until a wavelength becomes free on the output fibre. From that instant, the switch will transmit the remainder of the burst [15]. A burst that loses a portion while waiting for a free wavelength and whose remainder is successfully transmitted will henceforth be called a truncated burst.

We now introduce a simple analytical model to approximate the blocking probability of a single IP packet (rather than of a burst - since bursts can be fragmented and partially served) in a single isolated link with Poisson arrivals. Our approximation is asymptotically exact as the number of packets per burst approaches infinity.
To evaluate the packet loss probability in the case of a network, this approximation can replace the Erlang formula in (2). Note also that an exact solution for IP packet loss probability under the assumption that packets are exponentially distributed appears in [3]. In principle, the solution of [3] can also replace (2).

The underlying idea in our approach is to model the link by an $M / G / \infty$ queue (rather than by an $M / G / N / N$ queue). We will show that there is a one-to-one mapping between the states of the $M / G / \infty$ system and that of the Burst Segmentation system. Let $N$ be the number of available wavelength channels in the single link under consideration, and let $A$ be the traffic load offered to the $M / G / \infty$ system. The load $A$ equals the traffic load offered to the link under consideration.

The state of the $M / G / \infty$ queue is the number of busy servers denoted by $i$. Consider two cases of the state: (1) $0 \leq i \leq N$, and (2) $i>N$. In case (1), $i$ in the $M / G / \infty$ system is equivalent to the state of having $i$ wavelength channels busy in the Burst Segmentation system. In case (2), $i$ in the $M / G / \infty$ system is equivalent to the state of having $N$ wavelength channels busy in the Burst Segmentation system, and $i-N$ additional bursts that are losing packets. For example, if $i=N+1$, then one of the bursts is losing packets, as there is no means to buffer the packets in optical switching. However, once one of the $N$ servers (wavelength channels) becomes free, the remainder of that burst (that now becomes a truncated burst) is allocated to the free wavelength channel and immediately starts service.

Let $P(i)$ be the stationary probability that $i$ servers are busy in the $M / G / \infty$ system above. It is well known (see e.g., [2]) that the number of busy servers in an $M / G / \infty$ model has a Poisson distribution with parameter $A$. Thus,

$$
P(i)=A^{i} \frac{e^{-A}}{i !}, i=1,2, \ldots
$$

Notice that if $i>N$, then the rate of workload lost at state $i$ is $i-N$. The mean loss rate, denoted by $E[L]$, is therefore

$$
E[L]=\sum_{j=1}^{\infty} j \cdot P(k+j)=E\left[X_{A}-k\right]^{+},
$$

where $X_{A}$ is a Poisson random variable with mean $A$ and $[Z]^{+}$equals $Z$ if $Z>0$ and 0 otherwise.

The fraction of workload lost out of the total offered workload is then:

$$
B=\frac{E[L]}{A}
$$

The value of $B$ is used to approximate the packet loss probability which is asymptotically exact as the number of packets per burst approaches infinity. To find the blocking probabilities in an OBS network with Burst Segmentation, we simply replace (2) by (5).

\section{Route-dependent Priorities}

Preemptive priority burst service policies in each switching node that depends on the burst route [19] can reduce the overall blocking probability. It can also achieve other performance 
measures based on e.g., routes and fairness. Here we show how to evaluate the burst blocking probabilities when such policies are used.

For any route $\boldsymbol{r}$ and link $j$, let $h(\boldsymbol{r})$ be the number of hops of route $\boldsymbol{r}$, and $t(\boldsymbol{r}, j)$ be the number of hops of route $\boldsymbol{r}$ until and including link $j$. A route-dependent service priority at link $j$ could be any one-to-one mapping from the set of pairs $\{(h(\boldsymbol{r}), t(\boldsymbol{r}, j))\}$ into an ordered set of priority indices. The lower the priority index, the higher its service priority. Since we consider only static routes (that do not depend on the network state), any such route-dependent policy determines a static preemptive priority service policy at each link. Note that a given burst may be mapped into a different priority in each link along its route.

For a given isolated link where the offered loads of bursts from priority classes $p$ arrive as independent Poisson processes with rates $\rho^{p}, p \in \mathcal{P}=\{1,2, \ldots, P\}$, the burst blocking probabilities have been derived in [16]. Denote by $B_{j}^{p}$ the blocking probability of a burst from priority $p$ in link $j$, and by $p(\boldsymbol{r}, j)$ the priority of a burst from route $\boldsymbol{r}$ in link $j$. These blocking probabilities are expressed only as a function of $\rho^{p}$, $p \in \mathcal{P}$.

Under the blocking independence assumption above it follows that the reduced load of bursts from route $\boldsymbol{r}$ offered to link $j, \rho_{j}(\boldsymbol{r})$, satisfies

$$
\rho_{j}(\boldsymbol{r})=\mu_{j}^{-1} \lambda \boldsymbol{r} \prod_{i=1}^{J}\left(1-I(i, j, \boldsymbol{r}) \cdot B_{i}^{p(\boldsymbol{r}, i)}\right) .
$$

For each link $j$, let $\rho_{j}^{p}$ be the reduced load of bursts from priority $p$ arriving to link $j$. Observe that the set of reduced loads $\left\{\rho_{j}(\boldsymbol{r}), p(\boldsymbol{r}, j) \mid \boldsymbol{r} \in \mathcal{R}\right\}$ arriving to link $j$, determines the set $\left\{\rho_{j}^{p} \mid p \in \mathcal{P}\right\}$. Therefore, the set of new blocking probabilities $\left\{B_{j}^{p} \mid p \in \mathcal{P}\right\}$ can be computed from the reduced loads. The successive substitution iterations for these priority policies becomes similar to the case without priorities (derived in Section II) after replacing each $\rho_{j}$ and $B_{j}$ with the vectors $\boldsymbol{\rho}_{j}=\left(\rho_{j}^{1}, \ldots, \rho_{j}^{P}\right)$ and $\boldsymbol{B}_{j}=\left(B_{j}^{1}, \ldots, B_{j}^{P}\right)$, respectively.

\section{FiXed Point Approximation VAlidation}

To evaluate the quality of our approximation we simulate the Markov process $\left\{\left(X_{\boldsymbol{r}}(t), \boldsymbol{Y}_{\boldsymbol{r}}(t)\right) \mid \boldsymbol{r} \in \mathcal{R}, t \geq 0\right\}$ as described in Section II but without the blocking independence assumption. As emphasized in that section, we suggest that the blocking independence assumption is the key assumption that needs validation. The overhead introduced by the reservation headers and trailers is marginal and can easily be adjusted if needed.

Also, to get a fast simulator engine we re-sample the transmission time in every link along the route rather than sampling it once upon the burst arrival. To test this re-sampling approach and the impact of neglecting the reservation overhead, we compared the blocking probabilities obtained by our simulator with those obtained by a simulator that does not re-sample in every link and simulate the reservation mechanism. The difference observed in a four node network with JET was insignificant.

We use the NSFNET backbone network topology depicted in Figure 1 as a test bed to verify our reduced load fixed point approximation against simulation results.

The network topology comprises 13 OBS switches labelled $1,2, \ldots 13$, and 32 unidirectional fibre links, each comprising of 8 wavelength channels. For this test bed experiment we evaluate the blocking probabilities of 12 routes defined in Table I. The selected routes represent a variety of path lengths, link sharing degrees and mixtures of external and on-route internal traffic processes. All routes are shortest paths, except for $R_{3}$ and $R_{7}$ that are selected to obtain better route diversity.

For the verification process we consider symmetric exogenous arrival streams, where each route is offered the same exogenous rate of bursts. To represent a network with low, medium and high loads we consider three different arrival rates $\lambda=50,100,150$ bursts/sec for each route. The wavelength channel capacity and the burst length are set to yield a wavelength channel service rate $\mu=25$ bursts/sec.

Our objective is to validate our approximation against simulation for the JET policy, the Burst Segmentation policy and the following Route-Dependent priority policy, referred to as Least Remaining Hop-count First (LRHF). With LRHF, in every wavelength channel, each transmitted burst is preempted by any newly arrived burst that has a strictly less remaining number of hops to its destination. Another priority policy is also evaluated in Section V. The approximation and the simulation results are presented in Tables II and III.

Table II validates the approximation for the overall blocking probability using low, medium and high traffic loads for the JET, the LRHF priority and for the Burst Segmentation policies. The results are consistent with simulation.

Table III validates the approximation for the JET policy for each route and for the overall blocking probability using low, medium and high traffic loads. We observe that the simulation results agree very well with the approximation.

Thus in general, it seems that the error introduced by the blocking independence assumption is small. Statistically significant simulation results for the stationary blocking probabilities for each route under the priority and the segmentation policies requires extremely long runs for a network of this size, and they will be reported in the future.

\section{Performance Comparison}

In Table IV and Figures 2, 3, 4 and 5 we present the route and overall blocking probabilities obtained by our approximation method under the policies JET, Burst Segmentation (OCBS), LRHF and the following Most Traversed Hop-count First (MTHF) priority scheme. With MTHF, bursts that have traversed the most number of hops have the highest priority. This policy intends to protect bursts that have already used up significant network resources; whereas LRHF intends to protect bursts that are expected to use less network resources. Table IV presents the approximate probabilities for three 
different arrival rates, whereas Figures 2, 3, 4 and 5 depict them for a wider range of arrival rates.

From Figures 2, 3, 4 and 5 one may observe the following:

- From Figure 5, Burst Segmentation policy has the lowest overall blocking probability, while all others have similar values that are higher. Furthermore, in the range between low to medium loads, Burst Segmentation policy is significantly better than the other policies.

- From Figure 5 and Table IV, both priority policies improve the overall blocking probability of the JET policy, but not significantly. Between the two priority policies, LRHF performs better.

- As expected, the MTHF policy improves the blocking probabilities of long routes provided that their prefixes do not collide with higher or equal priority routes (see ROUTE 1 in Figure 2). If their prefixes do collide with higher or equal priority routes, then it still improves their blocking probabilities, but less than the improvement provided by the Burst Segmentation policy (see ROUTE 7 in Figure 3).

- As expected, the LRHF policy has an effect similar to that of the MTHF policy, but on short routes. That is, the blocking probabilities of short routes are reduced provided that their suffixes do not collide with higher or equal priority routes (see ROUTE 10 in 4). If their suffixes do collide with higher or equal priority routes, then it still improves their blocking probabilities, but less than the improvement provided by the Burst Segmentation policy (see ROUTE 4 in Figure 2).

\section{CONCLUSION}

We have developed a new and unified reduced load fixed point approximation model to evaluate the blocking probabilities of various policies in an OBS network. In particular, we have considered JET, Burst Segmentation and two priority schemes. The accuracy of the approximation was verified by simulations of the NSFNET network.

The numerical results have demonstrated the following phenomena: (1) Burst Segmentation policy has the lowest overall blocking probability, while the others are similar. (2) Both priority policies improve the overall blocking probability of the JET policy, but not significantly. (3) LRHF is better than MTHF with respect to the overall blocking probability. (4) The LRHF and MTHF priority policies can be used for service differentiation between long and short routes.

\section{ACKNOWLEDGMENT}

This work was supported by the Australian Research Council. The authors are also indebted to Eric W. M. Wong of City University of Hong Kong for his helpful comments.

\section{REFERENCES}

[1] R. A. Barry and P. A. Humblet, "Models of blocking probability in alloptical networks with and without wavelength changes", Proc., INFOCOM'95, pp. 4a.1.1-4a.1.11, 1995.

[2] D. Bertsekas and R. Gallager, Data Networks, $2^{\text {nd }}$ Edition, Prentice Hall, 1992.
[3] A. Detti, V. Eramo and M. Listanti, "Performance evaluation of a new technique for IP support in a WDM optical network: optical composite burst switching (OCBS)", IEEE Journal of Lightwave Technology, vol. 20, issue 2, pp. 154-165, Feb. 2002.

[4] K. Dolzer, C. Gauger, J. Spath and S. Bodamer, "Evaluation of reservation mechanisms for optical burst switching", AEU Int. J. of Electron. and Commun., vol. 55, no. 1, 2001.

[5] D. G., Foursa et al.," $2.56 \mathrm{~Tb} / \mathrm{s}(256 \times 10 \mathrm{~Gb} / \mathrm{s})$ transmission over 11,000 $\mathrm{km}$ using hybrid Raman/EDFAs with $80 \mathrm{~nm}$ continuous bandwidth", Proc. OFC 2002, Anaheim, Cal., pp. FC3 1-3, March, 2002.

[6] C. M. Gallep and E. Conforti, "Reduction of Semiconductor Optical Amplifier Switching Times by Preimpulse Step-Injected Current Technique", IEEE Photon. Technol. Lett., vol. 14 pp902-904, July 2002.

[7] F. P. Kelly, "Blocking probabilities in large circuit-switched networks", Adv. in Appl. Probab., vol. 18, pp. 473-505, 1986.

[8] G. M. Louth, Stochastic Networks: Complexity, Dependence and Routing, Ph.D dissertation, Univ. Cambridge, 1990.

[9] J. R. Munkres, Elements of Algebraic Topology, Perseus Press, p. 117, 1993.

[10] C. Qiao, "Labeled Optical Burst Switching for IP-over-WDM Integration”, IEEE Communications Magazine, pp. 104-114, Sept. 2000.

[11] R. Ramaswami and K. N. Sivarajan, "Optical Networks", 2nd Ed., Morgan Kaufmann, San Francisco, USA, 2002

[12] L. Rau et al., "Two-hop all-optical label swapping with varaible length $80 \mathrm{~Gb} / \mathrm{s}$ packets and $10 \mathrm{~Gb} / \mathrm{s}$ labels using nonlinear fiber wavelength converters, unicast/multicast output and a single EAM for 80 - to $10 \mathrm{~Gb} / \mathrm{s}$ packet multiplexing", Proceeding of OFC 2002, Anaheim, Cal., pp. DF2 1-3, March, 2002.

[13] Z. Rosberg, H. L. Vu and M. Zukerman, "Performance Evaluation for Optical Burst Switching Networks with Limited Wavelength Conversion", Proc. of the 7th IFIP Working Conference on Optical Network Design and Modeling, Budapest, Hungary, Feb. 2003.

[14] K. W. Ross, Multiservice loss models for broadband telecommunication networks, Springer-Verlag London Limited, 1995.

[15] V. M. Vokkarane, J. P. Jue and S. Sitaraman, "Burst segmentation: an approach for reducing packet loss in optical burst switched networks", in Proc. of ICC'02, Apr. 2002.

[16] H. L. Vu and M. Zukerman, Blocking probability for priority classes in optical burst switching networks, IEEE Communications Letters, vol. 6., no. 5, pp. 214-216, May 2002.

[17] D. Wada et al.,"Multi-hop 40 Gbits/s variable length photonic packet routing based on multi-wavelength label switching, waveband routing, and label swapping", Proceeding of OFC 2002, Anaheim, Cal., pp. 216217, March, 2002.

[18] J. Y. Wei, J. L. Pastor, R. S Ramamurthy and Y. Tsai, "Just-in-time optical burst switching for multi-wavelength networks", Proceedings of the 5th Int. Conf. on Broadband Commun. (BC'99), pp. 339-352, 1999.

[19] J. A. White, R. S. Tucker and K. Long, "Merit-based scheduling algorithm for optical burst switching, Proceedings of COIN-PS 2002, Cheju Island, Korea, July 2002.

[20] W. Whitt, "Blocking when service is required from several facilities simultaneously", AT\&T Technical Journal, vol. 64, no. 8, pp. 1807-1856, Oct. 1985.

[21] J. Yates, J. Lacey, D. Everitt and M. Summerfield, "Limited-Range Wavelength Translation in All-Optical Networks", proceeding of INFOCOM'96, pp. 954-961, 1996.

[22] J. Yu, K. Kojima, N. Chand, A. Ougazzaden, C. W. Lentz, J.M. Freund, and B. Mason, "Simultaneous Demultiplexing and Clock Recovery of $80 \mathrm{~Gb} / \mathrm{s}$ OTDM Signals Using a Tandem Electro-Absorption Modulator", 14th Annual Meeting of the IEEE Lasers and Electro-Optics Society., San Diego, USA, November 2001. 


\begin{tabular}{|c||c|c|c|c||c|c|c|c||c|c|c|c||}
\hline \multicolumn{1}{|c||}{ Routes $\backslash \lambda$} & \multicolumn{9}{|c||}{50} & \multicolumn{4}{c||}{100} & \multicolumn{4}{c||}{150} \\
\hline & JET & Seg. & LRHF & MTHF & JET & Seg. & LRHF & MTHF & JET & Seg. & LRHF & MTHF \\
\hline$R_{1}$ & 0.151 & 0.064 & 0.172 & 0.048 & 0.501 & 0.381 & 0.472 & 0.242 & 0.696 & 0.600 & 0.634 & 0.424 \\
\hline$R_{2}$ & 0.154 & 0.065 & 0.033 & 0.028 & 0.522 & 0.402 & 0.264 & 0.187 & 0.720 & 0.638 & 0.479 & 0.365 \\
\hline$R_{3}$ & 0.069 & 0.022 & 0.075 & 0.072 & 0.356 & 0.225 & 0.340 & 0.280 & 0.552 & 0.442 & 0.551 & 0.457 \\
\hline$R_{4}$ & 0.052 & 0.016 & 0.050 & 0.105 & 0.313 & 0.195 & 0.302 & 0.540 & 0.491 & 0.406 & 0.479 & 0.748 \\
\hline$R_{5}$ & 0.165 & 0.07 & 0.289 & 0.304 & 0.506 & 0.396 & 0.609 & 0.722 & 0.663 & 0.590 & 0.690 & 0.842 \\
\hline$R_{6}$ & 0.050 & 0.015 & 0.063 & 0.077 & 0.283 & 0.175 & 0.317 & 0.404 & 0.460 & 0.361 & 0.487 & 0.587 \\
\hline$R_{7}$ & 0.163 & 0.066 & 0.147 & 0.105 & 0.548 & 0.431 & 0.613 & 0.493 & 0.725 & 0.662 & 0.798 & 0.683 \\
\hline$R_{8}$ & 0.153 & 0.065 & 0.227 & 0.310 & 0.485 & 0.384 & 0.639 & 0.712 & 0.640 & 0.583 & 0.802 & 0.838 \\
\hline$R_{9}$ & 0.082 & 0.024 & 0.032 & 0.032 & 0.444 & 0.294 & 0.255 & 0.255 & 0.663 & 0.563 & 0.448 & 0.448 \\
\hline$R_{10}$ & 0.050 & 0.015 & 0.003 & 0.041 & 0.284 & 0.173 & 0.079 & 0.223 & 0.472 & 0.361 & 0.245 & 0.394 \\
\hline$R_{11}$ & 0.180 & 0.073 & 0.104 & 0.104 & 0.587 & 0.463 & 0.467 & 0.467 & 0.759 & 0.693 & 0.639 & 0.639 \\
\hline$R_{12}$ & 0.059 & 0.017 & 0.032 & 0.032 & 0.383 & 0.241 & 0.255 & 0.255 & 0.612 & 0.510 & 0.448 & 0.448 \\
\hline Overall & 0.111 & 0.043 & 0.102 & 0.105 & 0.434 & 0.313 & 0.384 & 0.398 & 0.621 & 0.534 & 0.558 & 0.573 \\
\hline
\end{tabular}

TABLE IV

ROUTE BLOCKING PROBABILITIES - APPROXIMATION.

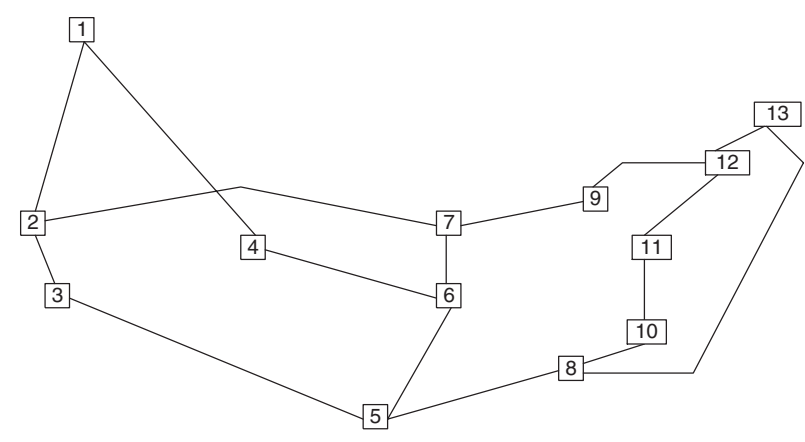

Fig. 1. NSFNET backbone network (T3, 45 Mb/s, April 1995).

\begin{tabular}{|c||l|}
\hline Route Name & Route hops \\
\hline$R_{1}$ & $1 \rightarrow 4 \rightarrow 6 \rightarrow 5 \rightarrow 8 \rightarrow 10$ \\
\hline$R_{2}$ & $2 \rightarrow 3 \rightarrow 5 \rightarrow 6 \rightarrow 7$ \\
\hline$R_{3}$ & $2 \rightarrow 7 \rightarrow 9 \rightarrow 12 \rightarrow 13$ \\
\hline$R_{4}$ & $3 \rightarrow 5 \rightarrow 8 \rightarrow 13$ \\
\hline$R_{5}$ & $5 \rightarrow 6 \rightarrow 7 \rightarrow 9 \rightarrow 12$ \\
\hline$R_{6}$ & $8 \rightarrow 10 \rightarrow 11 \rightarrow 12 \rightarrow 13$ \\
\hline$R_{7}$ & $10 \rightarrow 8 \rightarrow 5 \rightarrow 6 \rightarrow 4 \rightarrow 1$ \\
\hline$R_{8}$ & $7 \rightarrow 6 \rightarrow 5 \rightarrow 3 \rightarrow 2$ \\
\hline$R_{9}$ & $13 \rightarrow 12 \rightarrow 9 \rightarrow 7 \rightarrow 2$ \\
\hline$R_{10}$ & $13 \rightarrow 8 \rightarrow 5 \rightarrow 3$ \\
\hline$R_{11}$ & $12 \rightarrow 9 \rightarrow 7 \rightarrow 6 \rightarrow 5$ \\
\hline$R_{12}$ & $13 \rightarrow 12 \rightarrow 11 \rightarrow 10 \rightarrow 8$ \\
\hline
\end{tabular}

TABLE I

NSFNET ROUTES

\begin{tabular}{|c||c|c||c|c||c|c||}
\hline \multicolumn{1}{|c||}{ Routes $\backslash \lambda$} & \multicolumn{2}{c||}{50} & \multicolumn{2}{c||}{100} & \multicolumn{2}{c|}{150} \\
\hline & Approx. & Sim. & Approx. & Sim. & Approx. & Sim. \\
\hline$R_{1}$ & 0.151 & 0.142 & 0.501 & 0.485 & 0.696 & 0.684 \\
\hline$R_{2}$ & 0.154 & 0.153 & 0.522 & 0.516 & 0.720 & 0.713 \\
\hline$R_{3}$ & 0.069 & 0.068 & 0.356 & 0.347 & 0.552 & 0.546 \\
\hline$R_{4}$ & 0.052 & 0.050 & 0.313 & 0.307 & 0.491 & 0.491 \\
\hline$R_{5}$ & 0.165 & 0.161 & 0.506 & 0.501 & 0.663 & 0.659 \\
\hline$R_{6}$ & 0.050 & 0.049 & 0.283 & 0.280 & 0.460 & 0.456 \\
\hline$R_{7}$ & 0.163 & 0.162 & 0.548 & 0.545 & 0.725 & 0.723 \\
\hline$R_{8}$ & 0.153 & 0.143 & 0.485 & 0.475 & 0.640 & 0.636 \\
\hline$R_{9}$ & 0.082 & 0.075 & 0.444 & 0.428 & 0.663 & 0.652 \\
\hline$R_{10}$ & 0.050 & 0.047 & 0.284 & 0.274 & 0.472 & 0.460 \\
\hline$R_{11}$ & 0.180 & 0.166 & 0.587 & 0.568 & 0.759 & 0.750 \\
\hline$R_{12}$ & 0.059 & 0.057 & 0.383 & 0.378 & 0.612 & 0.607 \\
\hline Overall & 0.111 & 0.106 & 0.434 & 0.425 & 0.621 & 0.614 \\
\hline
\end{tabular}

TABLE III

Route Blocking PRoBAbILITIES WITH JET - APPROX. AND SimUl.

\begin{tabular}{|c||c|c||c|c||c|c||}
\hline \multicolumn{1}{|c||}{ Policy $\backslash \lambda$} & \multicolumn{2}{c||}{50} & \multicolumn{2}{c||}{100} & \multicolumn{2}{c|}{150} \\
\hline & Approx. & Sim. & Approx. & Sim. & Approx. & Sim. \\
\hline JET & 0.111 & 0.106 & 0.434 & 0.425 & 0.621 & 0.614 \\
\hline LRHF & 0.102 & 0.105 & 0.384 & 0.417 & 0.558 & 0.605 \\
\hline OCBS & 0.043 & 0.042 & 0.313 & 0.322 & 0.534 & 0.562 \\
\hline
\end{tabular}

TABLE II

OVERALL BLOCKING PROBABILITIES - APPROX. AND SIMUL. 

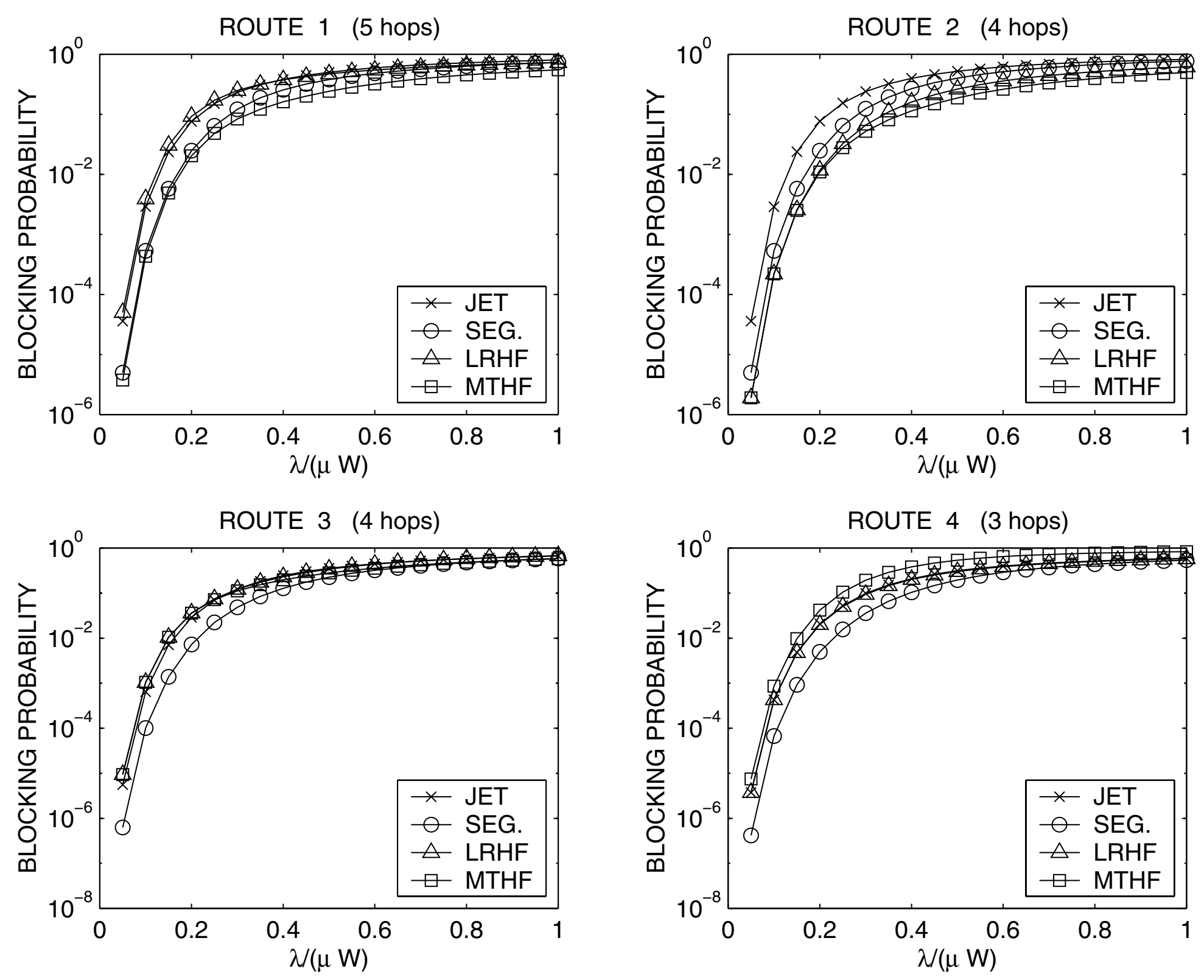

Fig. 2. Route blocking probabilities using JET, Seg., LRHF and MTHF policies 

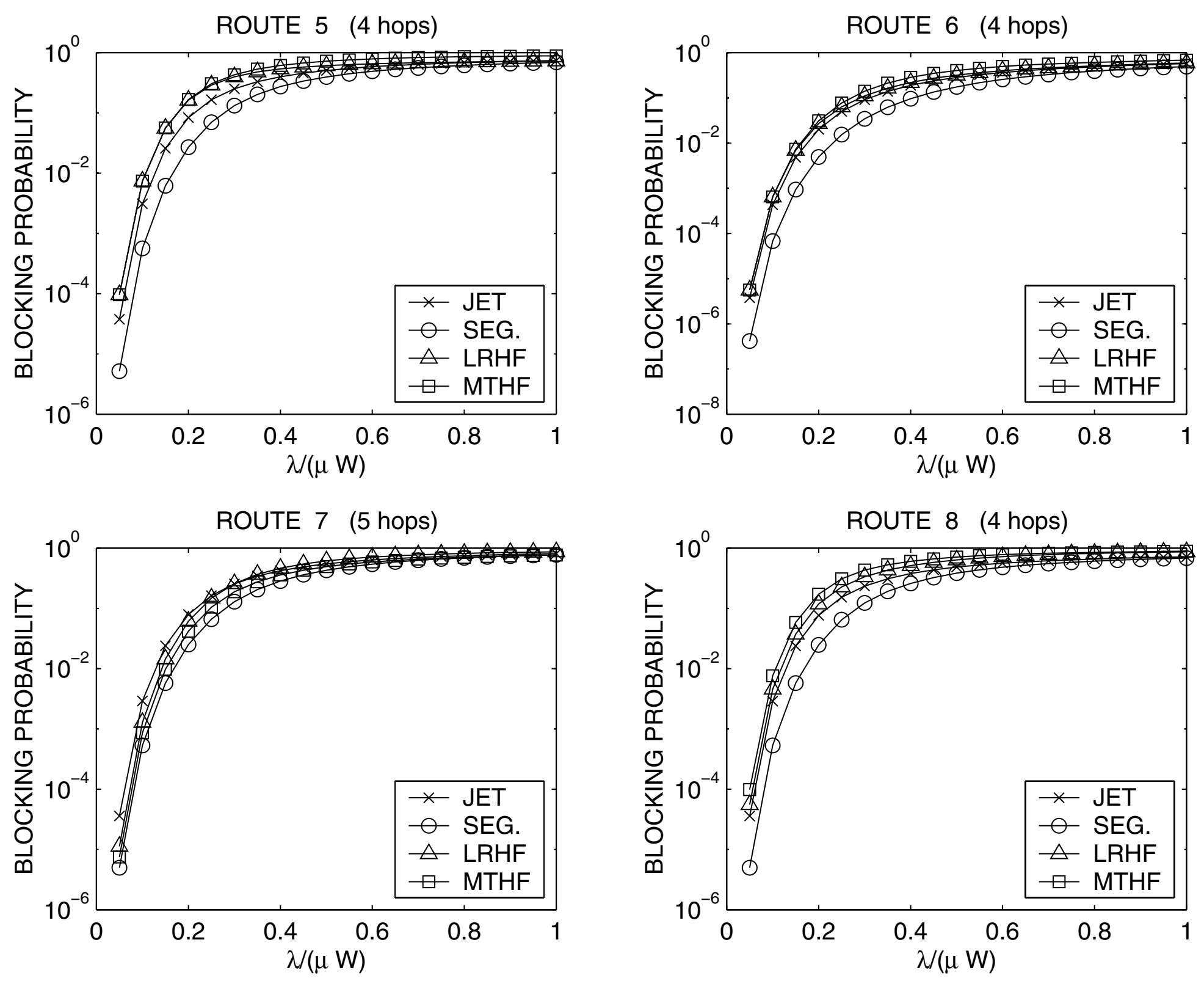

Fig. 3. Route blocking probabilities using JET, Seg., LRHF and MTHF policies 

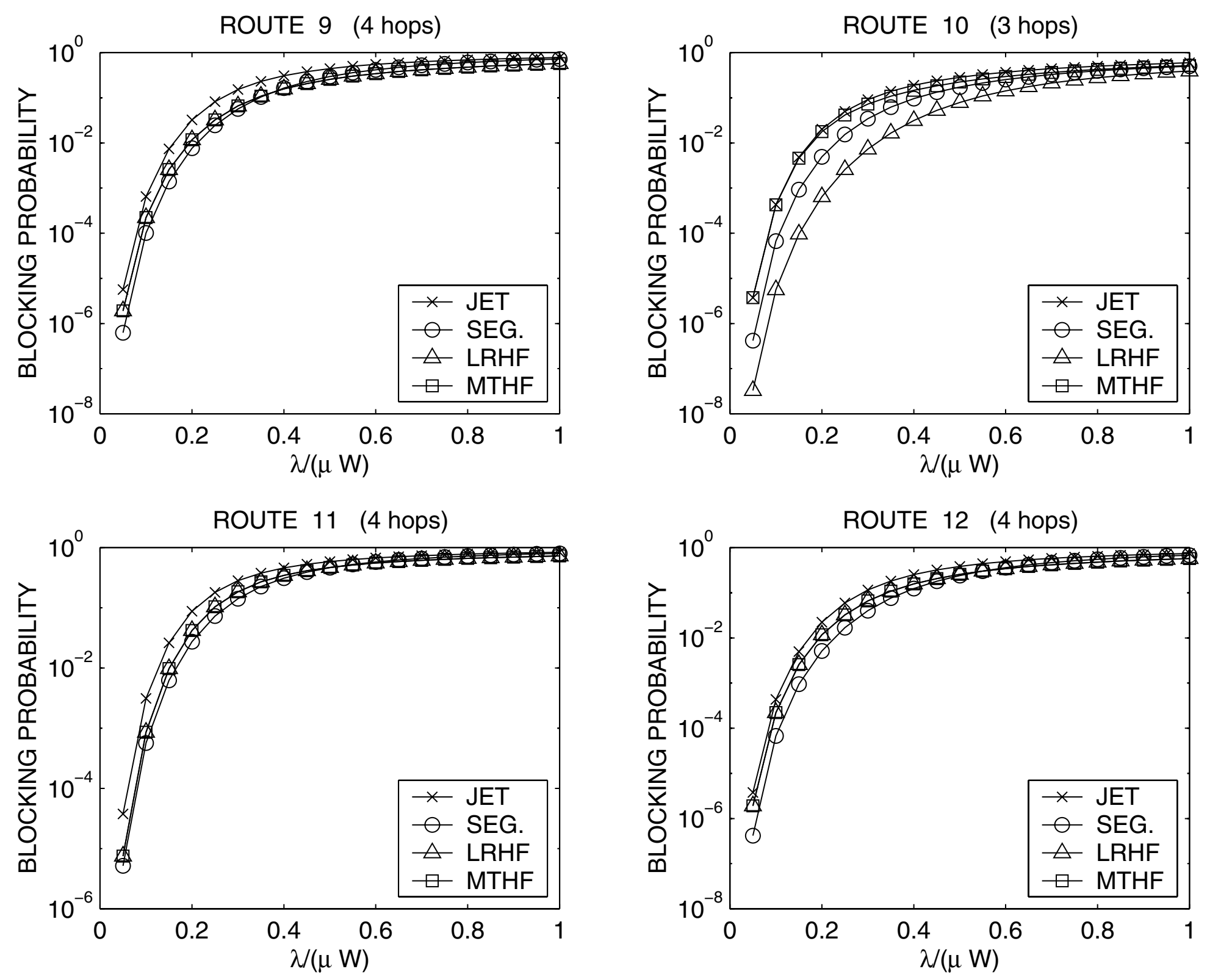

Fig. 4. Route blocking probabilities using JET, Seg., LRHF and MTHF policies 


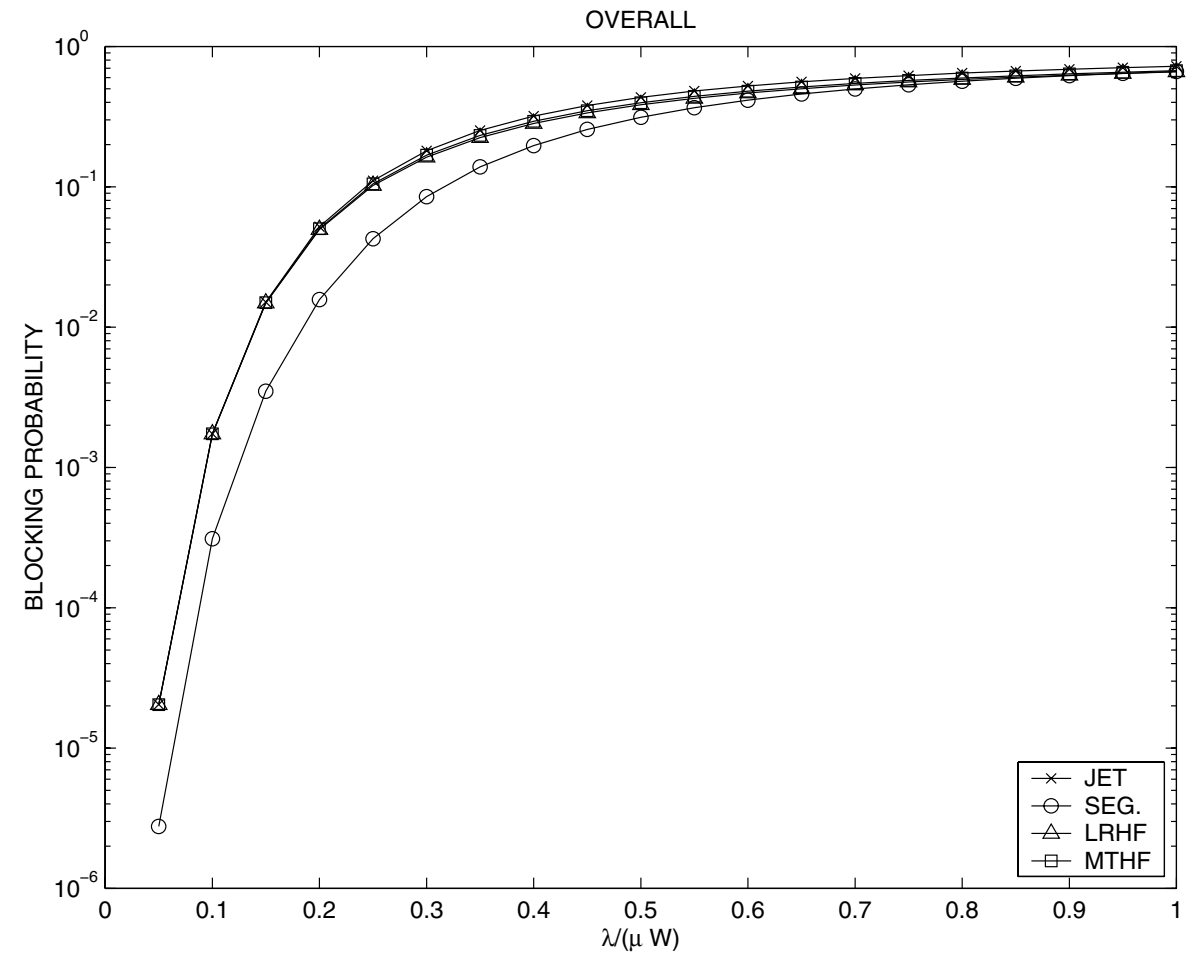

Fig. 5. Overall blocking probabilities using JET, Seg., LRHF and MTHF policies 